\title{
ESTUDIOS
}

\section{LAS DIFICULTADES EN EL PROCESO DE DECISIÓN VOCACIONAL EN RELACIÓN CON: EL GÉNERO, EL CURSO ACADÉMICO Y LOS INTERESES PROFESIONALES}

\author{
CAREER DECISION-MAKING DIFFICULTIES IN RELATIONSHIP WITH: \\ GENDER, ACADEMIC LEVEL AND CAREER INTEREST
}

\author{
Sara Lozano* y Elvira Repetto \\ Universidad Nacional de Educación a Distancia
}

\begin{abstract}
RESUMEN
El objetivo de este estudio radica en profundizar sobre el comportamiento vocacional de los jóvenes madrileños de entre 14 y 18 años. A través de análisis estadísticos como la T de Student y ANOVAs realizamos una evaluación del tipo y grado de dificultades que experimentan los jóvenes en el desarrollo de su proceso de decisión vocacional, y analizamos las diferencias existentes entre éstas en función del género, el curso académico y los intereses profesionales. Para ello se ha realizado la adaptación y validación de una escala basada en la teoría del procesamiento de la información denominada Escala de Dificultades en la Toma de Decisión de la Carrera. Tras los análisis realizados observamos que en líneas generales se confirman las hipótesis planteadas ampliando el marco de la investigación en Orientación Vocacional. Finalmente se presentan las conclusiones, limitaciones e implicaciones del estudio.
\end{abstract}

Palabras clave: Dificultades en el proceso de toma de decisión de la carrera, indecisión vocacional, estudio comparativo, diagnóstico en orientación profesional, enfoque del procesamiento de la información en la carrera, variables culturales.

\section{ABSTRACT}

The purpose of this research is to deepen on the vocational behaviour of 2.783 Spanish students aged between 14 and 18. Through statistical analysis like T Student and ANOVA, we analyze the difficulties that this sample experiments, and we investigate if it experiments different kinds or levels of dif-

\footnotetext{
* Correspondencia con las autoras: Dra. Sara Lozano Santiago, Universidad Nacional de Educación a Distancia, Facultad de Educación, Departamento de Métodos de Investigación y Diagnóstico en Educación II. Líneas de investigación: Orientación para el desarrollo de competencias socio-emocionales y Orientación Profesional. E-mail: slozano@edu.uned.es.
} 
ficulties based on variables like gender, academic grade, and professional interests. To do that we have adapted and validated a Career Decision-Making Questionnaire based on Processing Career Information Theory. We have confirmed the hypothesis raised, and we thus extend Vocational Guidance researches. Finally, implications for counselling and future research are also discussed.

Key words: Career decision-making difficulties, career indecision, comparative study, career guidance assessment, career processing information theory, cultural variables.

\section{Introducción}

Uno de los constructos que despierta más atención entre los investigadores de la Orientación Profesional es la "indecisión vocacional". Las miradas se dirigen hacia la percepción de las circunstancias que pueden impedir el curso normal de un proceso decisional, así como a la definición de sus características y del origen de dicha situación.

Tradicionalmente la indecisión ha sido observada como un problema puntual y desde una perspectiva negativa. Actualmente, no sólo se considera como una fase más en el proceso de desarrollo (frente a un estado) sino que, además, desde el marco que proyectamos nuestra investigación no se enfoca como algo negativo, sino como un elemento intrínseco que forma parte del proceso decisional. Es interesante comprobar cómo, a lo largo de la literatura y de las investigaciones, se intenta explicar esta realidad a través también de estudios causales. Desde hace unas décadas la técnica multivariante de los Modelos de Ecuaciones Estructurales se ha incorporado en el ámbito de la Orientación Profesional. Precisamente, a través de este tipo de estudios, podemos llegar a validar empíricamente algunas de las relaciones que se hipotetizan sobre el comportamiento vocacional. La escala, Career Decision-Making Difficulties Questionarie (Cuestionario de dificultades en la toma de decisión de la carrera) (CDDQ) de Gati y col. (1996) es uno de los instrumentos más utilizados en la última década y ha dado muestras de sus características y su utilidad tanto práctica como empírica (p.e. Mau, 2004; Gati y col., 2001; Gati y col, 1996 „Mau, 2001, Gati y Saka, 2001a, 2001b; Lancaster y col, 1999).

En España, Lozano (2004) realiza su adaptación y validación al castellano para la muestra de estudiantes comprendidos entre los 14 y 18 años en Madrid. Como ejemplo de la trascendencia de este instrumento, a continuación analizaremos con profundidad algunos de los estudios más recientes en los que se utiliza para profundizar sobre el comportamiento vocacional.

En primer lugar, Gati y Saka (2001a) tiene como objetivo analizar el comportamiento de la escala, Career Decision Difficulties Questionaire, $(C D D Q)$ en función de tres situaciones de elección académica de los estudiantes: Elección de la escuela superior en $9^{\circ}$ grado, elección de los cursos en $10^{\circ}$ grado, y decidirse por la opción militar en el $11^{\circ}$ grado. En cuanto a las diferencias en función del sexo, los chicos muestran más dificultades que las chicas en todos los grados en función de los "conflictos externos" y las "creencias disfuncionales" y en otras categorías como la forma de obtener información, "conflictos internos", en general se encontraron diferencias obteniendo puntuaciones más altas los varones.

En segundo lugar, Albion y Fogarty (2002) validan y comparan la estructura de CDDQ en dos muestras mediante el modelo de Ecuaciones estructurales. Una de ellas está compuesta por 121 estudiantes de secundaria mientras que en el segundo participan adultos. En líneas generales en el estudio se confirma la multidimensionalidad de la estructura de 
CDDQ, ajustando mejor cinco factores que tres, y el ajuste del modelo sugiere en ambas muestras la misma estructura, mientras que observamos que los adultos muestran menos dificultades que los jóvenes.

A continuación presentaremos los modelos presentados por los autores, tanto para el modelo de medida (Fig. 1) como la relación con las variables de: personalidad, intereses, inteligencia y género (Fig. 2).

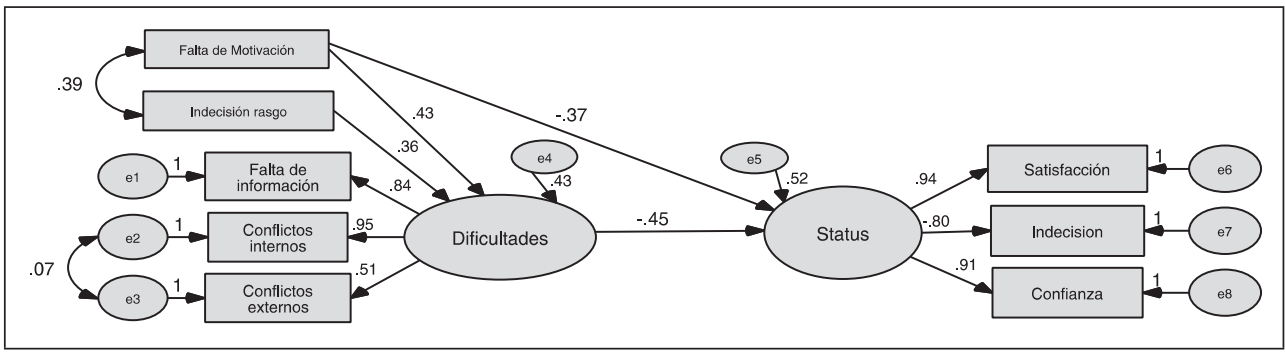

FIGURA 1.

Modelo estructural del cuestionario CDDQ (Albion y Fogarty, 2002, p. 106).

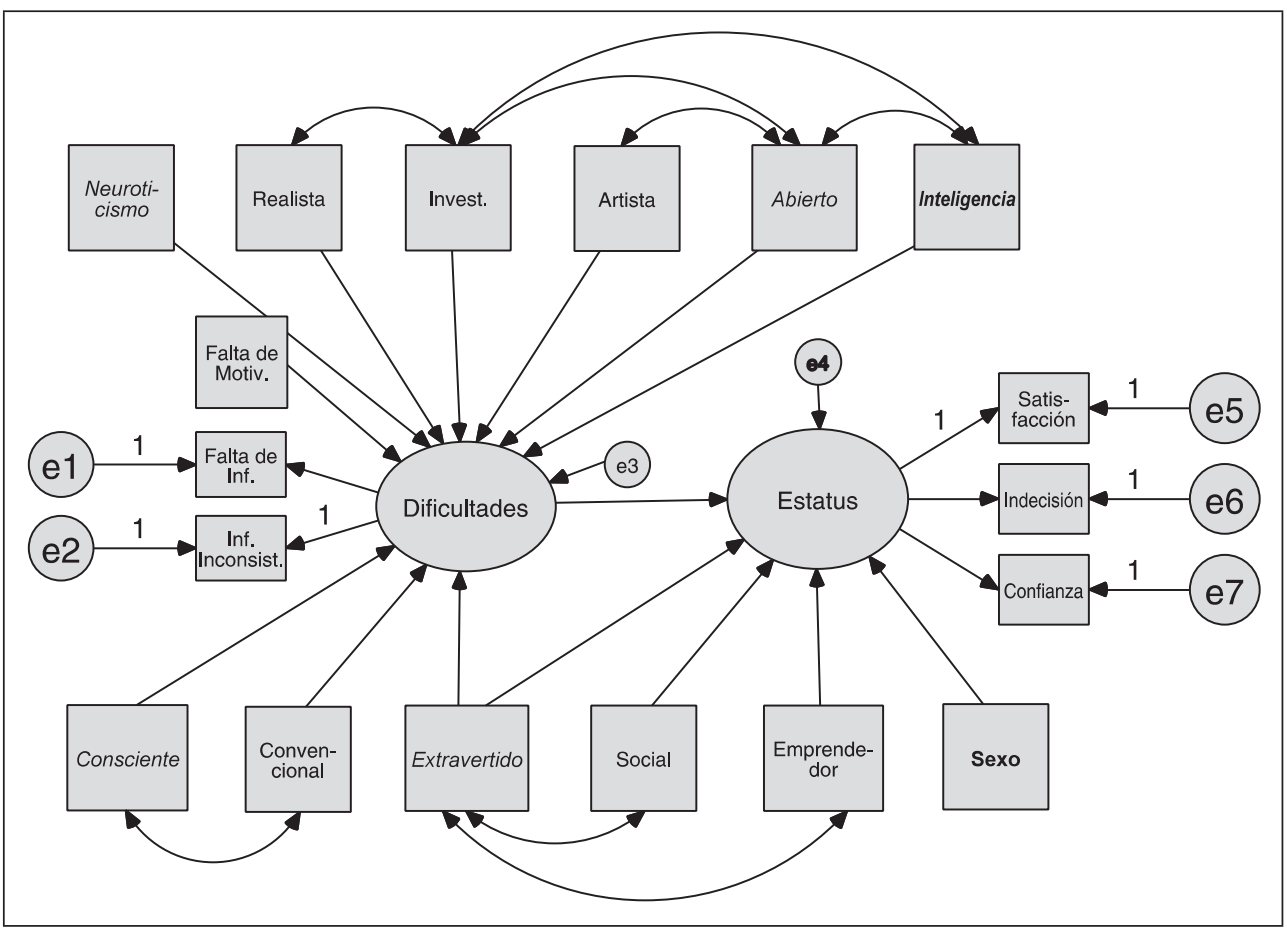

FIGURA 2.

Modelo conceptual del proceso de decisión de la carrera (Albion y Fogarty, 2002, p. 96). 
La variable latente sobre las dificultades esta presentada como una variable compuesta de influencias multidireccionales. Se observan las diferencias entre las variables en función de la personalidad, intereses e inteligencia. La variable status esta conformada por varios ítems previos a la escala en los que se les pregunta por su satisfacción y su nivel de indecisión y de confianza.

En la muestra de Albion y Fogarty (2002) el 70\% de la muestra se mostraban como muy indecisos, pero no se mostraban especialmente preocupados por dicha indecisión. Las puntuaciones más bajas (escala tipo likert con un rango de respuesta de 1 a 5) se encontraron en los conflictos externos $(2,43)$ y falta de motivación $(2,89)$, y las más altas en los mitos disfuncionales $(4,88$ en escala de 9$)$, indecisión $(4,38)$ y las cuatro escalas de indecisión $(3,88-4,08)$.

MacCallum y Browne (1993) presentan un estudio similar donde ellos definen un modelo identificando algunas de sus variables latentes, no como constructos definidos por variables manifiestas, sino como variables compuestas, las cuales resultan de una combinación lineal de indicadores causuales observados. Antes de evaluar el modelo, se realizo los estudios correlaciones, aunque AMOS utiliza análisis de covarianza, Hoyle y Panter (1995) recomiendan realizar correlaciones que indican las relaciones básicas entre los elementos del modelo. El estudio provee de apoyo a los hallazgos también encontrados en otras investigaciones sobre la relación significativa entre los intereses y la personalidad. También se hallan dicha relacen significativas entre los intereses profesionales y las dificultades; siendo los intereses convencionales los que se relacionan positivamente con la falta de información y la información inconsistente. Aquellos cuya área vocacional preferente era la "Investigación" mostraban menos dificultades generales y especialmente de información inconsistente; mientras que los sujetos con intereses "Sociales" mostraban más problemas en relación con la categoría de falta de información.

Por último, en uno de los estudios más recientes, Lease (2004) nos muestra el efecto del las variables de los sistemas atribucionales, la información laboral y el mentoring sobre las dificultades en la decisión de la carrera. Como podemos observar se trata de una medida de gran actualidad y que nos permite ampliar el campo de investigación. De esta suerte, Lozano (2004) adapta y valida este instrumento de Gati et al. (1996) para la muestra española.

En esta investigación nos planteamos el objetivo de analizar el comportamiento de una muestra de estudiantes, en función de una serie de variables descriptivas en relación con las dificultades que estos experimentan en su proceso de desarrollo de la carrera. Partiendo de la variable independiente de las Dificultades en la Toma de Decisión de la Carrera (DTDC), se considera analizar si existen diferencias significativas o no en ese tipo de dificultades en función del género, nivel educativo, el estatus académico de la familia, los intereses profesionales y la situación vocacional del sujeto. Tratándose de un estudio exploratorio para la población española, podemos anticipar que los estudios con otras poblaciones apuntan a la formulación de las siguientes hipótesis direccionales:

- Hipótesis 1: Tanto hombres como mujeres experimentan el mismo nivel de dificultades a la hora de realizar un proceso de decisión vocacional.

- Hipótesis 2: Los estudiantes que cursan el primer año de su Etapa Educativa presentan más dificultades en su proceso de decisión vocacional que los que están finalizando su etapa. 
- Hipótesis 3: Los sujetos con intereses vocacionales cercanos al campo profesional del área social experimentan mayor nivel de dificultad al realizar su proceso de decisión vocacional.

\section{Metodología}

\section{Muestra}

La muestra del estudio está conformada por un total de 2.783 alumnos de Madrid, de los cuales el $42,5 \%$ son varones y el $57,5 \%$ son mujeres. En función del Nivel Educativo, el $62,2 \%$ de los sujetos se encuentran en el Segundo Ciclo de ESO, dicha etapa se corresponde con uno de los "momentos críticos" en la toma de decisión profesional de los adolescentes. El 32,6\% cursan Bachillerato, en este nivel educativo el alumno ya ha definido en parte la opción académica sobre la que quiere ir avanzando en su opción profesional; aunque sin duda las posibilidades posteriores son amplias (Opciones Universitarias, de Ciclos Formativos de Grado Superior, Escuelas Profesionales, etc). Un 4,7\% de los alumnos están cursando un Ciclo Formativo de Grado Medio (opción profesional definida) y un 0,5\% se encuentran realizando un Programa de Garantía Social.

En relación a la edad, la distribución de la muestra se ajusta a los niveles educativos descriptos, comprobando que tan sólo un 5,1\% tienen más de 19 años, el 67,9\% se encuentra entre los 14-16 años y el 27\% entre los $17-18$ años.

En función del país de origen, observamos como el 95,2\% de nuestra muestra son españoles. El resto de la muestra se distribuye entorno a 47 países de origen, de los cuales el $2,9 \%$ de la muestra proceden de países latino-americanos.

Por último, otra de las variables identificadoras seleccionadas para este estudio corresponde a las Áreas Vocacionales Preferentes de los alumnos. A pesar del gran número de valores perdidos y alumnos que obviaron señalar cuál era su área vocacional preferente (el " $n$ " total asciende a 668 alumnos), sí podemos observar cómo las Área Vocacionales más elegidas son (en orden descendente según las frecuencias) las presentadas en la tabla 1. Las áreas que obtienen mayores porcentajes son las referidas al campo de la "investigación científica" $(14,8 \%)$ y "técnica" $(11,0 \%)$ así como el de la "Sanidad" $(10,6 \%)$, seguido de la Técnica $(7,7 \%)$ y Comunicación $(7,1 \%)$. En relación a la opción vocacional preferentes, el $57 \%$ de los alumnos reconocen que el área elegida se encuentra entre sus 2 o 3 opciones preferidas, el 22,6\% reconoce que es su única opción preferida mientras que el 15,8\% tan sólo reconoce que le gusta y un 3,5\% que tiene dudas sobre si es esa su opción preferida o no. De otro modo, resulta interesante comprobar que el $85 \%$ aproximadamente de los alumnos considera "probable" y con "seguridad" que desarrolle su profesión dentro del área que ha elegido, mientras que el $15 \%$ restante considera que tiene pocas o ninguna posibilidad de ejercer su profesión dentro del área vocacional que ha elegido.

\section{Instrumentos}

Escala sobre Dificultades en el la Toma de Decisión e la Carrera (E-DTDC) (Lozano, 2004). La escala lo que pretende es evaluar en qué áreas y qué factores están interfiriendo 
TABLA 1: Distribución de la muestra según el área vocacional preferente.

\begin{tabular}{|c|c|c|c|}
\hline \multicolumn{4}{|c|}{ ÁREA VOCACIONAL PREFERENTE (CIBAP) } \\
\hline & & Frecuencia & $\begin{array}{l}\text { Porcentaje } \\
\text { válido }\end{array}$ \\
\hline $\begin{array}{l}\text { Perdidos } \\
\text { Total }\end{array}$ & $\begin{array}{l}\text { Investigación Técnica } \\
\text { Investigación Científica } \\
\text { Sanidad } \\
\text { Técnica aplicada } \\
\text { Comunicación } \\
\text { Deportes } \\
\text { Enseñanza y orientación } \\
\text { Protección y seguridad } \\
\text { Economía y negocios } \\
\text { Música } \\
\text { Cine y teatro } \\
\text { Actividades Administrativas } \\
\text { Artes plásticas } \\
\text { Estética } \\
\text { Humanístico-Social } \\
\text { Derecho y Asesoramiento } \\
\text { Atención personal } \\
\text { Idiomas } \\
\text { Actividad Agropecuaria } \\
\text { Total } \\
0\end{array}$ & $\begin{array}{r}313 \\
233 \\
225 \\
163 \\
151 \\
146 \\
134 \\
120 \\
103 \\
81 \\
75 \\
71 \\
61 \\
54 \\
53 \\
49 \\
48 \\
26 \\
9 \\
2.115 \\
668 \\
2.783\end{array}$ & $\begin{array}{r}14,8 \\
11,0 \\
10,6 \\
7,7 \\
7,1 \\
6,9 \\
6,3 \\
5,7 \\
4,9 \\
3,8 \\
3,5 \\
3,4 \\
2,9 \\
2,6 \\
2,5 \\
2,3 \\
2,3 \\
1,2 \\
0,4 \\
100,0\end{array}$ \\
\hline
\end{tabular}

de manera más significativa en los procesos de decisión académico-profesional en los alumnos, con la finalidad de diseñar un plan de intervención adecuado a sus necesidades. Se trata de la adaptación de Career Decision-Making Difficulties Questionarie (CDDQ) (Gati, I., Krausz, y Osipow, S. H., 1996). Una escala tipo Likert de 27 item, con un intervalo de respuesta de a 1 a 7 , en donde 1 significa que la persona no se identifica con la situación descrita en el item (no tiene dificultades) y 7 que se identifica completamente (tiene muchas dificultades). La estructura de la escala la presentamos en la tabla 2.

TABLA 2: Taxonomía de la Escala de Dificultades en la Toma de Decisión de la Carrera.

\begin{tabular}{|l|l|l|}
\hline $\begin{array}{l}\text { Dificultades antes del proceso } \\
\text { de toma de decisión }\end{array}$ & $\begin{array}{l}\text { F3DTDC: Indecisión } \\
\text { F4DTDC: } \text { Motivación }\end{array}$ & \\
\hline \multirow{2}{*}{$\begin{array}{l}\text { Dificultades durante el proceso } \\
\text { de toma de decisión }\end{array}$} & Falta de información & $\begin{array}{l}\text { F1DTDC: Académico profesional } \\
\text { F2DTDC: Sobre uno mismo }\end{array}$ \\
\cline { 2 - 3 } & Resolución de conflictos & $\begin{array}{l}\text { F5DTDC: Conflictos internos } \\
\text { F6DTDC: Conflictos externos }\end{array}$ \\
\hline
\end{tabular}


En la tabla 2 nos limitamos a señalar las subescalas de la prueba y su estructura que fue confirmada también a través de un modelo de medida con bondades de ajustes muy satisfactorias $(\mathrm{CFI}=.997, \mathrm{GFI}=.988)$. En este modelo (utilizado en el modelo final de este estudio) los parámetros gamma alcanzan uno valores altos de 0,96 y de 0,85 y al igual que en los modelos anteriores todos los parámetros son significativos confirmando la hipótesis teórica que se plantea de la relación como antecedente de la indecisión y la falta de motivación sobre las otras categorías de dificultades focalizadas durante el propio proceso de la toma de decisión. El alpha de Cronbach de la escala con una muestra de 1.060 sujetos es de 0,91 .

Cuestionario de Intereses Básicos Académico-Profesionales - CIBAP- (Hernández, 2001), Área vocacional preferente. A través de esta variable nuestro objetivo es el de tener información sobre los intereses académico-profesionales de nuestros alumnos, a través de la reflexión de los alumnos en cuanto a sus Áreas vocacionales preferentes. Operativamante estas áreas las medimos a partir del "Cuestionario de Intereses Básicos Académico-Profesionales —CIBAP__” (Hernández, 2001), cuyo objetivo no sólo nos permite contar con una información fiable sobre los intereses básicos de nuestra muestras, sino que también se trata de una herramienta que pretende informar a los estudiantes sobre el contenido de cada una de las 19 Áreas de Intereses Básicos que define —a través de posibles estudios universitarios, ciclos formativos y profesionales características de cada área-.

\section{Procedimiento}

La población está referida a los estudiantes de la Comunidad de Madrid que cursan, según el MECD (curso 2002-2003) del Segundo Ciclo de Educación Secundaria Obligatoria 93.470 sujetos, Ciclos Formativos de Grado Medio, 20.560 y Bachillerato 20.560, y programa de Garantía Social 6.749. El tipo de muestreo fue incidental, se invitaron a participar en este proyecto a 98 centros educativos de los cuales participaron finalmente 18. La administración de las pruebas se llevó a cabo por tutores después de haber recibido una sesión formativa y la duración fue de una sesión de una hora.

Se han realizado análisis descriptivos de la población en todas las variables y posteriormente para los estudios comparativos utilizaremos la técnica del la T de Student y el análisis de varianza, que nos permite analizar las diferencias entre más de dos grupos. Para ello utilizaremos las variables control como criterio para diferenciar a los grupos y analizar si existen diferencias significativas en cuanto al tipo de dificultades que experimentan la técnica de la T de Student y la ANOVA tomando como valores de referencia de la significatividad una probabilidad de error de .001. A su vez se han realizado estudios post-hoc basados en la técnica de Tukey y así comprobar la magnitud y valor de las diferencias.

\section{Resultados}

En primer lugar evaluamos las diferencias en función del sexo (Hipótesis 1). Teniendo en consideración los diferentes tipos de dificultades que evaluamos con la escala podemos afirmar que no se encuentran diferencias significativas entre hombres y mujeres en cuanto a su percepción de los conflictos internos $(\mathrm{p}=0,94, \mathrm{t}=0,71)$, y si asumimos una $\mathrm{p}<0,01$ 
tampoco se encontrarían en cuanto a la falta de información sobre uno mismo $(\mathrm{p}=0,042$, $\mathrm{t}=-2,03)$ y sobre el mundo académico-profesional $(\mathrm{p}=0,24, \mathrm{t}=-2,256)$. En el resto de las subescalas, es decir, en las dificultades referidas a falta de motivación $(p=0,000, t=4,397)$, de indecisión $(\mathrm{p}=0,001, \mathrm{t}=-3,452)$, y conflictos externos $(\mathrm{p}=0,000, \mathrm{t}=4,91)$, sí existen diferencias significativas, pudiendo comprobar que las mujeres experimentan menos dificultades en relación a la "motivación" y "conflictos externos", mientras que los hombres presentan valores más bajos en cuanto a sus dificultades referidas a la "indecisión".

En relación con el curso académico (Hipótesis 2) los resultados son bastante contundentes, estableciendo diferencias significativas en todas las categorías de dificultades, hallando en todas las categorías $\mathrm{p}=0,000$, exceptuando en la subescala sobre la indecisión con una $\mathrm{p}=0,016(\mathrm{~F}=2,781)$. En cuanto a los análisis post-hoc (Tukey) comprobamos varios aspectos que pasamos a exponer en función del grupo de dificultades implicado (Figura 3):

- Inseguridad en uno mismo y falta de motivación. De una forma significativa destacan los alumnos de $3^{\circ}$ de ESO con mayores dificultades a este respecto que el resto de alumnos. Sin duda más que una dificultad podemos entender que se trata de un proceso normal dado el momento en el que se encuentra en su proceso decisional. De esta forma los resultados nos ayudan a confirmar que los alumnos de cursos más bajos, a los que se les exige menor toma de decisión se sienten más inseguros y desmotivados para enfrentarse a estas decisiones. Sin embargo también resulta interesante comprobar que los alumnos (a pesar del escaso número) de PGS parecen presentar más dificultad que los de $3^{\circ}$, lo cual también nos viene a informar del perfil de estos alumnos, menos motivados y con unas experiencias de aprendizaje que les pueden situar en cierta desventaja ante la toma de decisión sobre su futuro.

- Falta de Información sobre uno mismo. En este grupo de dificultades sucede algo parecido al anterior, sin embargo en este caso los alumnos de $4^{\circ}$ de ESO parecen presentar más dificultades que los de $3^{\circ}$. La reflexión que hacemos a este respecto iría en la línea de que el grado de conciencia que los alumnos también van tomando sobre la realidad de la decisión y de uno mismo va siendo mayor conforme van creciendo. Conforme se nos van planteando retos vamos descubriendo cuáles son nuestras carencias. No cabe duda que parecen ser los alumnos tanto de $4^{\circ}$ de ESO como los de Garantía Social son los que pueden reclamar más información sobre su personalidad y sus habilidades para tomar decisiones profesionales.

- Falta de información sobre las opciones académico-profesionales. En cuanto a esta categoría sí consideramos interesante destacar que tanto los alumnos de Bachillerato como los de Ciclos Formativos de Grado Medio se sitúan con medias por debajo del resto de los alumnos.

- Conflictos internos y las dificultades ante alternativas (F5DTDC). En este caso, son los alumnos de CFGM los que se sitúan significativamente con menos dificultades en esta categoría, por encima de los de $3^{\circ}, 4^{\circ}$ y PGS. Resulta coherente, dado que estos alumnos ya han realizado una elección académico-profesional bastante concreta y las posibilidades de elección entre alternativas muy dispares se reduce.

- Conflictos externos. En esta categoría, parece que los alumnos que se encuentran cursando la opción de $1^{\circ}$ de Bachillerato son los que experimentan menos dificultades o presión por circunstancias externas ante sus decisiones. 


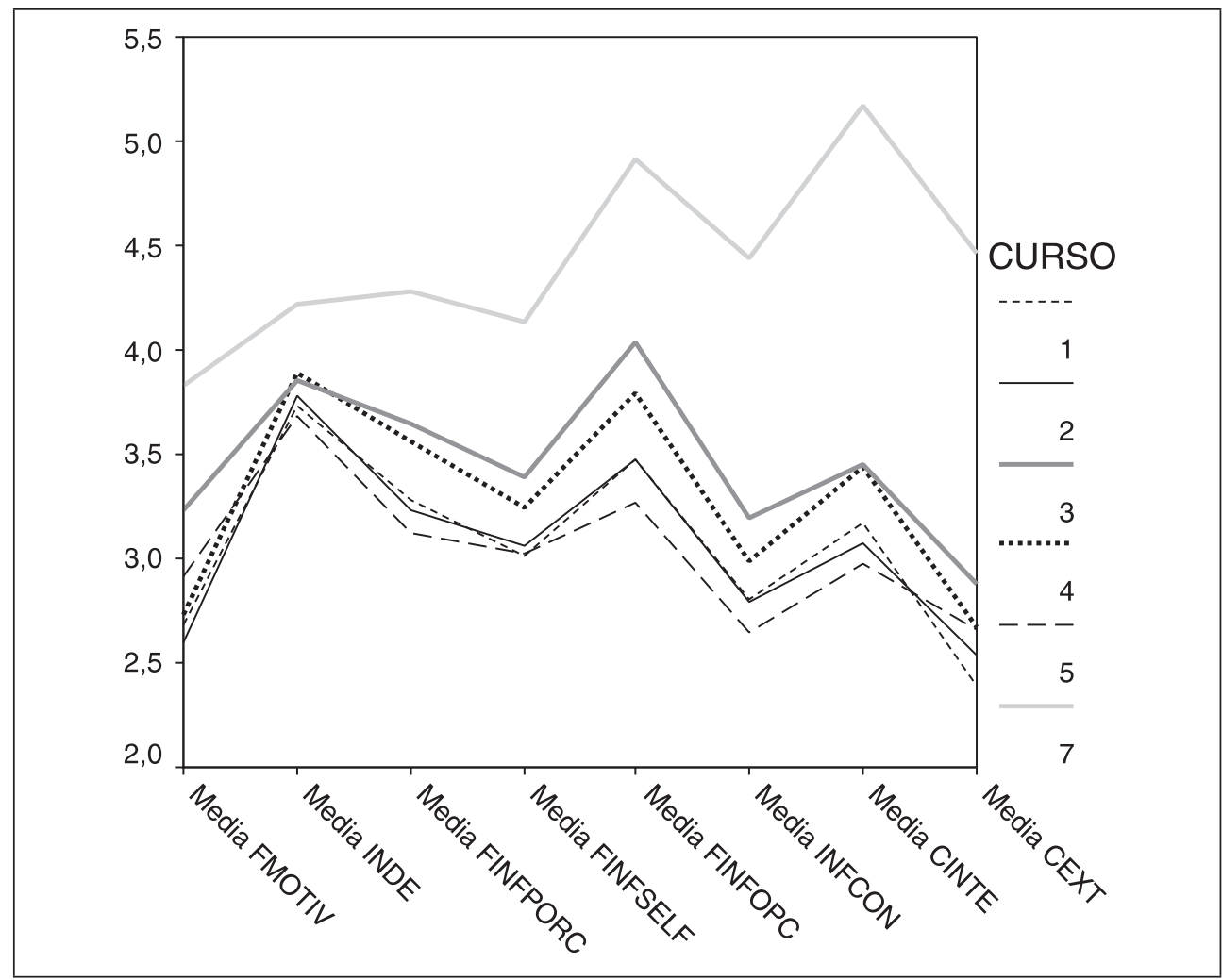

FIGURA 3.

Representación gráfica de las diferencias de medias en la E-DTDC en función del curso académico ( $1=1^{\circ}$ Bach.; 2 = $2^{\circ}$ Bach.; $3=3^{\circ} \mathrm{ESO} ; 4=4^{\circ} \mathrm{ESO} ; 5$ = CFGM; 7 = PGS).

En relación con las diferencias en DTDC en función de los intereses vocacionales en función del área vocacional que han escogido como preferente (Hipótesis 3), en la tabla 4 observamos que sí existen tales diferencias con valores en todas las categorías cuya probabilidad de error oscila entre 0,000 y 0,003 .

Sin embargo, cuando analizamos estos resultados de forma detallada mediante los estudios post-hoc comprobamos que estas diferencias no son muy altas, y que parecen poco significativas cuando se realizan las comparaciones múltiples. En líneas generales sí debemos destacar que alumnos que eligen la opción de Deporte suelen presentar mayor "Falta de motivación" ante el proceso de decisión así como experimentan de una forma más significativa "Conflictos externos". También podemos apuntar que los alumnos que eligen opciones como Sanidad, Música, Comunicación, etc., es decir opciones con salidas académicas más definidas o determinadas, frente a la opción de Investigación Científica, Aplicada, etc.; suelen experimentar menos dificultad a la hora de buscar información sobre sus opciones académicas, al contrario de lo que se había hipotetizado, mostrando menores dificultades alumnos de opciones profesionales "Sociales". 
TABLA 3: ANOVA de la escala de DTDC en función del área vocacional preferente.

\begin{tabular}{|c|c|c|c|c|c|c|}
\hline & & $\begin{array}{l}\text { Suma de } \\
\text { cuadrados }\end{array}$ & gl & $\begin{array}{c}\text { Media } \\
\text { cuadrática }\end{array}$ & $\mathbf{F}$ & Sig. \\
\hline $\begin{array}{l}\text { Inseguridad } \\
\text { Yo-Motivación }\end{array}$ & $\begin{array}{l}\text { Inter-grupos } \\
\text { Intra-grupos } \\
\text { Total }\end{array}$ & $\begin{array}{r}119,421 \\
3.943,543 \\
4.062,963\end{array}$ & $\begin{array}{r}18 \\
2.973 \\
2.091\end{array}$ & $\begin{array}{l}6,634 \\
1,902\end{array}$ & 3,488 & 0,000 \\
\hline $\begin{array}{l}\text { F. Inf. Proceso } \\
\text { TDC }\end{array}$ & $\begin{array}{l}\text { Inter-grupos } \\
\text { Intra-grupos } \\
\text { Total }\end{array}$ & $\begin{array}{r}70,040 \\
3.501,421 \\
3.571,461\end{array}$ & $\begin{array}{r}18 \\
2.076 \\
2.094\end{array}$ & $\begin{array}{l}3,891 \\
1,687\end{array}$ & 2,307 & 0,001 \\
\hline F. Inf. Yo mismo & $\begin{array}{l}\text { Inter-grupos } \\
\text { Intra-grupos } \\
\text { Total }\end{array}$ & $\begin{array}{r}102,339 \\
3.734,968 \\
3.837,308\end{array}$ & $\begin{array}{r}18 \\
2.077 \\
2.095\end{array}$ & $\begin{array}{l}5,686 \\
1,798\end{array}$ & 3,162 & 0,000 \\
\hline $\begin{array}{l}\text { F. Inf. Opciones } \\
\text { acd.-prof. }\end{array}$ & $\begin{array}{l}\text { Inter-grupos } \\
\text { Intra-grupos } \\
\text { Total }\end{array}$ & $\begin{array}{r}87,201 \\
4.583,483 \\
4.670,684\end{array}$ & $\begin{array}{r}18 \\
2.079 \\
2.097\end{array}$ & $\begin{array}{l}4,845 \\
2,205\end{array}$ & 2,197 & 0,003 \\
\hline $\begin{array}{l}\text { Conflictos } \\
\text { internos-Inf. } \\
\text { contradictoria }\end{array}$ & $\begin{array}{l}\text { Inter-grupos } \\
\text { Intra-grupos } \\
\text { Total }\end{array}$ & $\begin{array}{r}112,683 \\
5.287,535 \\
5.400,218\end{array}$ & $\begin{array}{r}18 \\
2.077 \\
2.095\end{array}$ & $\begin{array}{l}6,260 \\
2,546\end{array}$ & 2,459 & 0,001 \\
\hline $\begin{array}{l}\text { Conflictos } \\
\text { externo }\end{array}$ & $\begin{array}{l}\text { Inter-grupos } \\
\text { Intra-grupos } \\
\text { Total }\end{array}$ & $\begin{array}{r}135,678 \\
3.017,656 \\
3.153,335\end{array}$ & $\begin{array}{r}18 \\
2.075 \\
2.093\end{array}$ & $\begin{array}{l}7,538 \\
1,454\end{array}$ & 5,183 & 0,000 \\
\hline
\end{tabular}

\section{Conclusiones}

A lo largo del estudio hemos podido comprobar el comportamiento vocacional de nuestra muestra en cuanto a sus dificultades en el proceso de decisión profesional. Partiendo de la Escala de Dificultades en el proceso de Toma de Decisión de la Carrera (Lozano, 2004), en la que evalúa el nivel de "Falta de Motivación", "Falta de Información sobre uno mismo", "Falta de Información sobre las opciones académico-profesionales", "Falta de información sobre el proceso de decisión", "Dificultades ante conflictos internos", y "Dificultades ante conflictos externos", hemos podido comprobar si existen diferencias significativas en nuestra muestra en función de diferentes variables. En concreto, podemos advertir que las hipótesis planteadas se confirman si se considera un tratamiento global de la escala, y si analizamos de forma parcial observamos que existen variaciones en función de las dificultades estudiadas. De esta suerte, aunque no existen diferencias significativas en función del género en el nivel de dificultades, sí observamos tal y como se comprueba en otros estudios (p.e. Gati y Saka, 2001a), que las mujeres plantean menores dificultades a la hora de afrontar conflictos. En cuanto al nivel educativo, se observa que las diferencias radican, más que en la edad, en la situación del curso tal y como habíamos hipotetizado, observando menores dificultades en los alumnos de CFGM y de $2^{\circ}$ de Bachillerato. Resulta interesante observar que, aunque la muestra es muy pequeña, son los alumnos de Programas de Garantía Social los que plantean mayores dificultades. También es significativo que en $4^{\circ}$ de la ESO toman mayor conciencia y presentan más dificultades en cuanto a la categoría que engloba 
falta de información. En cuanto al estatus académico de la familia los resultados no confirman nuestra hipótesis, mientras que en relación con los intereses vocacionales lo confirma parcialmente. Dado que existe una muestra reducida para la realizar las comparaciones a este respecto, sí parecen existir diferencias significativas, aunque con magnitudes pequeñas. Además estas diferencias no favorecen a los alumnos que eligen enseñanzas técnicas, sino a aquellos que realizan elecciones de profesiones con salidas profesionales muy definidas, coincidiendo en parte con algunas profesiones del área Social como la Enseñanza y el Deporte.

Una de las limitaciones fundamentales de la investigación se refiere a las posibilidades de generalización de los resultados, dado que la muestra, aunque grande no resulta significativa. A su vez, son numerosos los estudios que se pueden realizar a partir de los datos y profundizar sobre cada uno de los aspectos, por lo que se abren diferentes puertas para continuar estudiando la relación de estas variables con otras nuevas.

Algunas de las implicaciones prácticas que se pueden derivar de esta investigación se refieren a la intervención psicopedagógica en el ámbito de la orientación profesional. A partir de la adaptación de la escala de Dificultades en el Proceso de Toma de Decisión de la Carrera (Lozano, 2004) podemos intervenir de forma diferenciada en función de las áreas o aspectos en los que el sujeto se muestre más deficitario, así como potenciar todos aquellas estrategias y actitudes que facilitan un proceso decisional sano. A la luz de los resultados, podemos intuir que en cuestiones relacionadas con las estrategias que se utilizan para tomar decisiones, no existen diferencias en función del género, y que por lo tanto hombres y mujeres han podido vivir experiencias de aprendizaje similares a este respecto, aún teniendo que prestar especial atención a las capacidades para afrontar conflictos de los varones y a la capacidad o posible sumisión de las mujeres. De otro modo, a través del estudio también confirmamos que es necesario iniciar los procesos de orientación desde los primeros cursos para así lograr decisiones más realistas y libres en aquellos cursos donde los alumnos deben tomar decisiones definitivas. Además parece relevante contemplar con mayor urgencia la orientación de alumnos en Programa de Garantía Social, que dadas sus características deberían estar muy orientados al mundo laboral y son los que parecen presentar mayores dificultades. En líneas generales, el orientador profesional tiene en su mano guiar los procesos de decisión vocacional facilitando experiencias de aprendizaje en este ámbito, y ayudado al sujeto a tomar conciencia de la gran variedad de elementos que van conformando sus decisiones académico-profesionales.

\section{Referencias bibliográficas}

Albion, M. J. y Fogaty, G. J. (2002). Factors influencing career decision making in adolescents and adults. Journal of Career Assessment, 10, 91-126.

Gati, I. y Saka, N. (2001a). High school students' career-related decision-making difficulties. Journal of Counseling and Development, 79, 331-340.

Gati, I. y Saka, N. (2001b). Internet-Based versus paper-and-pencil assessment: measuring Career Decision-Making Difficulties. Journal of Career Assessment, 9, 397-416

Gati, I., Krausz, M. y Osipow, S. H. (1996). A taxonomy of difficulties in career decision-making. Journal of Counseling Psychology, 43, 510-526. 
Gati, I., Osipow, S. H., Krausz, M. \& Saka, N. (2000). Validity of the Career Decision-Making Difficulties Questionnaire: counselee versus career counselor perceptions. Journal of Vocational Behavior, 56, 99-113.

Gati, I., Osipow, S. H., Krausz, M. \& Saka, N. (2000). Validity of the Career Decision-Making Difficulties Questionnaire: counselee versus career counselor perceptions. Journal of Vocational Behavior, 56, 99-113.

Hair, J. F. Jr., Anderson, R. E., Tatham, R. L. y Black, W. C. (1999) (5ª Ed. $1^{\text {a }}$ en español). Análisis Multivariante. Madrid: Prentice Hall Iberia.

Hernández, V. (2001). Análisis causal de los intereses profesionales en los estudiantes de Secundaria.. Tesis Doctoral pendiente de publicar. Universidad Complutense de Madrid.

Hoyle, R. H. y Panter, A. T. (1995). Writing about structural equation models. En R. H. Hoyle (Ed), Structural equation modeling: Concepts, issues, and applications (pp. 158-176). Thousand Oaks, CA: Sage.

Kalmar-Gome, T. (1999). Differences between those who seek career counseling and those who seek information in the career library, in the types and severity of their difficulties their interests' crystallisation and personality type. Tesina sin publicar, Univerity of Haifa, Israel.

Lancaster, P. L. Rudolph, C., Pekins, S. y Patten, T. (1999). The reliability and validity o the career decision difficulties questionnaire. Journal of Career Assessment, 7, 393-413.

Lease, S. H. (2004). Effect of locus of control, work knowledge, and mentoring on career decisionmaking difficulties: Testing the role of race and academic institution. Journal of Career Assessment, 12, 239-254.

Lozano, S. (2004). Validación de un modelo causal y de medida de la auto-eficacia y las dificultades en el proceso de decisión de la carrera profesional. Madrid: UNED (Tesis doctoral pendiente de publicación).

MacCallum, R. C. y Browne, M. W. (1993). Use of causal indicators in covariance structural modeling: Some practical issues. Psychological Bulletin, 114, 533-541.

Mau, W. C. (2000b). Assessing career decision-making difficulties: A cross-cultural study. Paper presented at the Annual Meeting of the ACA, Washington, DC.

Merenda, P. F. (1997). A guide to he proper use of factor analysis in the conduct and reporting of research: Pitfalls to avoid. Measurement and Evaluation in counseling and Development, 30, 156164.

Osipow, S. H. y Gati, I. (1998). Construct and concurrent validity of the Career Decision-Making Difficulties Questionnaire. Journal of Career Assessment, 6, 347-364

Osipow, S. H., Carney, C. G., Winer, J. L., Yanico, B. y Koschier, M. (1976b). The Career Decision Scale ( $3^{\text {rd }}$ ed). Columbus, OH: Marathon consulting and Press.

Osipow, S. H. (1987) Manual for Career Decision Scale. Lutz, FL: Psychological Assessment Resources, Inc.

Tabachnick, B. G. y Fidell, L. S. (1996). Using multivariate statistics. New York: HarperColling.

Visauta, B. (1986). Técnicas de investigación social: Modelos Causales. Barcelona: Hispano Europea. 